\title{
TITLE:
}

\section{Chronic Mountain Sickness}

$\operatorname{AUTHOR}(S)$ :

León-Velarde, Fabiola; Rivera, Maria; Garcia, Patricia J.

\section{CITATION:}

León-Velarde, Fabiola ...[et al]. Chronic Mountain Sickness. ヒマラヤ学 誌 2010, 11: 158-164

ISSUE DATE:

2010-05-01

URL:

https://doi.org/10.14989/HSM.11.158

RIGHT: 


\title{
Chronic Mountain Sickness
}

\author{
Fabiola León-Velarde $^{1)}$, Maria Rivera ${ }^{1)}$, Patricia J. Garcia ${ }^{2)}$ \\ 1) School of Sciences, Universidad Peruana Cayetano Heredia, Lima, Peru \\ 2) School of Public Health, Universidad Peruana Cayetano Heredia, Lima, Peru \\ Peru is an Andean country with populated cities over 3,500 meters above sea level. The Cayetano Heredia \\ University has been working in research of several aspects associated with high altitude. Chronic mountain \\ sickness (CMS) and High Altitude Pulmonary Hypertension (HAPH), as different entities or together in the same \\ subject, have been well established in the Andean region as well as in the Tibetan and Qinghai regions. We will \\ present briefly the existing data on clinical features, and pathophysiology of CMS, with emphasis on the findings \\ from the Andes. Excessive erythrocytosis is the outstanding sign of CMS, and right ventricular enlargement, \\ pulmonary hypertension, and remodeling of pulmonary arterioles are hallmarks of HAPH. Risk factors as, age, \\ chronic obstructive diseases, sleep disorders, obesity and menopause, increase the prevalence of these disorders. \\ CMS subjects also show higher erythropoietin concentration, lower oxygen saturation and hypoxic ventilatory \\ response; higher physiological dead space and higher $\mathrm{CO}_{2}$, suggesting ventilatory inefficiency. The smaller \\ vasodilatation to normobaric hypoxia and larger vasoconstriction to hypocapnia in CMS subjects, suggest that \\ control of vascular resistance and cerebrovascular responses may be impaired. Familial character and heritability \\ studies have suggested that genetic factors could make a contribution to the pathogenesis of CMS and HAPH.
}

\section{Introduction}

Chronic mountain sickness (CMS) affects people who are native or long time residents of high altitude. It is characterized by an excessive erythrocytosis for the altitude of residence, severe hypoxemia and in many cases, particularly in severe CMS, high altitude pulmonary hypertension (HAPH). CMS usually begins insidiously in adult life associated probably to aging; the clinical picture disappears when the patient moves to lower altitudes.

CMS and HAPH represent separate manifestations of chronic hypoxia, namely stimulation of erythropoiesis and stimulation of pulmonary hypertension, respectively. However, in many patients with severe CMS, both manifestations are present simultaneously ${ }^{1)}$. Thus, patients with moderate CMS may have little or no elevation of pulmonary artery pressure or resistance beyond the normal increase at high altitude ${ }^{2)}$. Alternatively, particularly in children and young adults, life-threatening HAPH after a relatively short exposure to altitude may occur, with little or no increase in hemoglobin $(\mathrm{Hb})$ concentration ${ }^{3,4)}$.
In 1925, a Peruvian renowned scientist, Dr. Carlos Monge presented the first description of $\mathrm{CMS}^{5)}$. This patient was a native of the mining town of Cerro de Pasco located at $4,300 \mathrm{~m}$ in the Peruvian Andes, working as an office employee. His symptoms disappeared with descent to Lima (sea level) but recurred upon returning to $4,300 \mathrm{~m}$. In 1928 Monge published an extensive article ${ }^{6)}$ on cases of CMS from Cerro de Pasco $(4,300 \mathrm{~m})$ and Puno $(3,800 \mathrm{~m})$, an agricultural town. The Dean of the Medical School of Paris suggested the name of Monge' s Disease for this clinical picture. Monge considered CMS to be a "loss of acclimatization" because it developed only after prolonged exposure to altitude in previously wellacclimatized subjects.

\section{Definition, symptoms and signs of CMS}

CMS is defined as a clinical syndrome characterized by excessive erythrocytosis (females $\mathrm{Hb}>19 \mathrm{~g} / \mathrm{dl}$; males $\mathrm{Hb}>21 \mathrm{~g} / \mathrm{dl}$ ), severe hypoxemia and in some cases moderate or severe pulmonary hypertension ${ }^{7)}$. This definition refers to "normal natives" or permanent 
residents who are assumed to have normal respiratory function. Usually starts near the fourth decade of life. CMS is more common in Andean natives than in Tibetans and it has not been reported in Ethiopian highlanders.

The most common symptoms of CMS are headaches, dizziness, dyspnea, sleep disturbances (insomnia, hypersomnia), tinnitus, physical and mental fatigue, alterations of memory, loss of appetite, and bone and muscle pain. Patients with CMS have usually intermittent or permanent cyanosis, venous dilatation in hands and feet, and clubbing of the fingers and toes. In advanced stages of the disease right heart failure secondary to excessive pulmonary hypertension usually appears ${ }^{6,8)}$. The laboratory findings show hemoglobin concentration and hematocrit, above the normal levels for the altitude of residence. The arterial $\mathrm{O}_{2}$ partial pressure $\left(\mathrm{PaO}_{2}\right)$, and the arterial $\mathrm{O}_{2}$ saturation $\left(\mathrm{SaO}_{2}\right)$ are lower, and the arterial $\mathrm{CO}_{2}$ partial pressure $\left(\mathrm{PaCO}_{2}\right)$ is higher than altitude normal values. Bicarbonate concentration is higher than the normal for the altitude of residence, indicating mild respiratory alkalosis with partial renal compensation $^{6 \sim 9)}$.

\section{Mechanisms of CMS}

The pathophysiological sequence of CMS can be understand as follows, a decrease of the ventilatory function at high altitude produces hypoxemia, which is at the basis of the excessive erythrocytosis.

Highland population from the Andes living above 3000 meters, have a blunted ventilatory response to increasing hypoxia, and are "breathless" compared to acclimatized newcomers but more compared to sea level natives at sea level. CMS patients are relatively hypoxic and hypercapnic when judged against healthy HA natives. Thus, CMS has been associated with poor hypoxic ventilatory responses (HVR), although there is no proof that alterations in carotid body physiology are the cause of hypoventilation in CMS. To better understand the peripheral chemoreceptor sensitivity to hypoxia, it has been usually employed acute and sustained protocols of exposure to hypoxia. An acute HVR (AHVR) protocol evaluates the acute response to a stepwise induction of hypoxia over few minutes. A sustained protocol (15-20 minutes of hypoxia) determines the hypoxic ventilatory decline (HVD), which is the attenuation of the initial ventilatory response after the first few minutes of hypoxia. AHVR reflects peripheral control of breathing, while the HVD may reflect attenuation of the VE response by central control mechanisms, although there is some controversy on this.

When SL and HA natives as well as patients with CMS are exposed acutely to 7 minutes of hypoxia, from an initial end-tidal $\mathrm{PO}_{2}\left(\mathrm{PETO}_{2}\right)$ of 100 Torr to a final $\mathrm{PETO}_{2}$ of 45 or 34 Torr, the average values for the slope of the AHVR for both HA natives and subjects with CMS are around one-third of the values for SL natives. HA natives and subjects with CMS do not differ significantly from each other in any of the variables studied. Overall, recent studies provide evidence to support the notion that the lower levels of ventilation in CMS subjects, i.e. the loss of the drive to breathe, may arise from mechanisms other than reductions in the peripheral chemoreflex sensitivity to hypoxia, or that its contribution is quite marginal.

Overall, our interpretation is that CMS subjects have reset their central $\mathrm{CO}_{2}$ chemoreceptors to operate around the resting $\mathrm{PETCO}_{2}$ close to sea level standards, around high $\mathrm{PETCO}_{2}$ values for high altitude. The central modulators of respiration (glutamate, an activator and GABA, a depressor), which decrease the response to $\mathrm{CO}_{2}$, show an exacerbated action in hypoxia. Hence, a counterbalanced role of these neuromodulators, directed to compensate the possibly reduced sensitivity of the respiratory center to $\mathrm{CO}_{2}$, could be hypothesized as a plausible explanation. However, with hypoventilation CSF also accumulates $\mathrm{HCO}_{3}{ }^{-}$. Thus a role for $\mathrm{HCO}_{3}{ }^{-}$in chemosensitive signaling, even if not extensively investigated, could be claimed minimizing the role of $\mathrm{CO}_{2}$ in control of breathing.

\section{CMS and age}

With normal aging, arterial oxygen pressure $\left(\mathrm{PaO}_{2}\right)$ decreases linearly, from about 95 Torr at the age of 20 to about 75 Torr at the age of $75^{10)}$. The hemoglobin- 
oxygen equilibrium curve is sigmoid in shape, and varies little with altitude. ${ }^{12)}$ Thus at sea level, $\mathrm{SaO}_{2}$ of $85 \%$ or more falls on a flat part of the OEC, so that small changes in $\mathrm{PaO}_{2}$ have little effect on saturation. However, at altitude, where $\mathrm{PaO}_{2}$ is lower, small changes in $\mathrm{PO}_{2}$ can have large effects on saturation and the same changes that accompany aging may have significant effects on arterial hemoglobin saturation and therefore arterial oxygen content $\left(\mathrm{CaO}_{2}\right)$.

Monge C. and Whittembury have shown, by combining the equation describing the $\mathrm{PaO}_{2}$ drop with age with the one describing the hemoglobin response to $\mathrm{PaO}_{2}$ in high altitude natives, that the increase in hematocrit at high altitude as a function of age can be predicted $^{11)}$. Based on the above physiologic observations and epidemiological studies, we proposed that at high altitude, as age increases, CMS will increase its prevalence. Figure 1 shows indirectly that age is an important contributory factor for the development of excessive erythrocytosis and CMS. It indicates that ventilation of Andean high altitude natives responds poorly to acute hypoxia and hyperoxia, but does respond to $\mathrm{CO}_{2}$ added to the inspired air. However, all these responses fall with age and, therefore, an appropriate response to $\mathrm{CO}_{2}$ does not mean that the ventilation will be independent of age.
Arai et al. ${ }^{12)}$ have shown that $\mathrm{PaCO}_{2}$ increases with age at altitudes between 3,450 to $3,850 \mathrm{~m}$., in other words ventilation decreases with age. Additionally, vital capacity of high altitude men decreases more with age than that of sea level men, together with a decrease in oxygen saturation. Observational studies have not shown a correlation between CMS and age, but they have been based in small sample of cases. The comparison of prevalences of people with CMS at different ages should be more likely to reveal the effect of aging on the prevalence of CMS in a certain population.

In addition to the role played by the age-related decrease in ventilation in the etiology of $\mathrm{CMS}^{13)}$, other additional factors may be involved in the development of CMS: an increase in the alveolar-arterial $\mathrm{O}_{2}$ gradient with possible venous-arterial shunting and a reduction of the ventilation-perfusion ratio ${ }^{14,15)}$.

\section{CMS in women}

Some years ago, CMS was considered an almost exclusively male condition, since little was known about its prevalence and associated risk factors in women. Pre-menstrual women have been thought to be protected from CMS because the female hormones, progestin and estrogen, increase alveolar ventilation

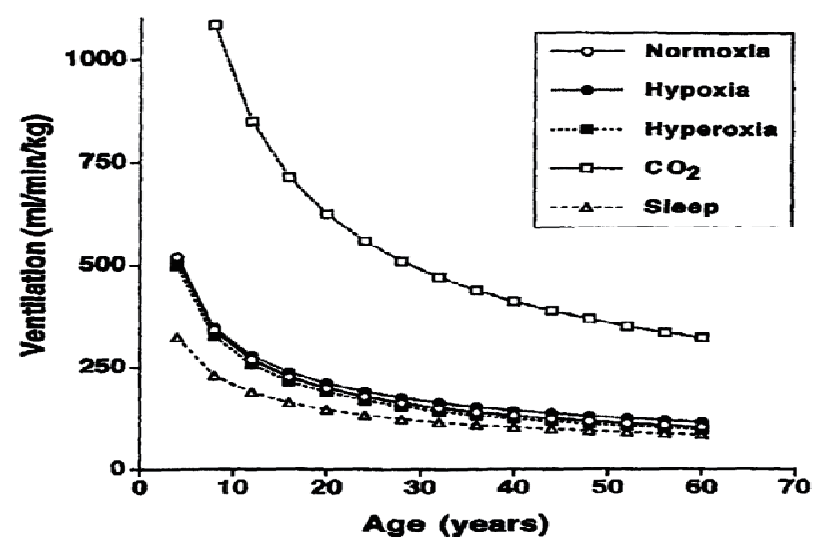

Figure 1: Age-related ventilation $\left(V_{e}\right)$ in high altitude natives residing at 4,500 m. $V_{e}$ was measured under conditions of normoxia, hypoxia, hyperoxia, with $\mathrm{CO}_{2}$ added to the inspired air, and during sleep. From ref ${ }^{11}$. 
and the hypoxic ventilatory response ${ }^{16)}$. In addition, having low concentrations of male hormones may inhibit erythropoiesis ${ }^{17)}$. Menstruation might act like a phlebotomy, protecting pre-menopausal women from developing excessive erythrocytosis. Unfortunately, no data have been available to shed light on these possibilities.

León-Velarde et al. ${ }^{18)}$ measured Hct, $\mathrm{SaO}_{2}$ and peak expiratory flow rates (PEFR) in pre- and postmenopausal high altitude women. After menopause women have higher Hct, lower $\mathrm{SaO}_{2}$ and lower PEFR. In addition, post-menopausal women have a significantly higher frequency of symptoms associated with CMS. The same group found that pre-menopausal women resident at high altitude have higher oxygenation and lower $\mathrm{Hb}$ levels, associated with higher levels of progesterone in the luteal phase of the cycle. Progesterone increases alveolar ventilation during pregnancy and the luteal phase of the menstrual cycle, and this effect is enhanced when combined with estrogen. The post-menopausal decrease of hormones could depress alveolar ventilation and $\mathrm{PaO}_{2}$, stimulate erythropoiesis, increase viscosity, decrease tissue perfusion and lead to further erythropoiesis and, ultimately, CMS.

Santolaya et al.19) have also shown in women with 55 years of age or older, that $\mathrm{PaO}_{2}$ values were below those of age-matched men living at the same altitude. Arterial $\mathrm{PCO}_{2}$ showed a sharp rise after age 40, reaching values similar to or above those of men. These observations support the notion that ventilatory drive in women has an important hormonal component, and that this stimulus decreases after menopause.

\section{CMS and metals}

Metals have been found to interact with different respiratory and hematopoietic metabolic pathways, thus the exposure to metals in high altitude natives could contribute to excessive erythrocytosis by aggravating hypoxemia or through other pathogenic mechanisms.

Contamination with heavy metals is considered a great occupational and environmental health problem, particularly in cities with high mining activity.
Extractive industries occupy a place of first order in the Andean economies. Bolivia, Chile and Peru are one of the main world producers of copper, silver, lead and zinc. The route of entrance of metals to the organism, their particle size and type (organic or inorganic), determine the concentration and the possibility of their diffusion towards the organism.

A preliminary study carried out in the city of Cerro de Pasco, a mining city (Villafuerte FC, Personal Communication), demonstrated that there were not differences in the $\mathrm{Pb}$ and $\mathrm{Cu}$ serum levels between healthy high altitude natives and subjects with CMS. Conversely, a strong positive correlation was found between the serum $\mathrm{Zn}$ concentration and hematocrit concentration in patients with excessive erythrocytosis.

Another study showed that $52 \%$ of patients with an hematocrit of at least $75 \%$ had raised serum cobalt (Co) concentrations (range 22-71 [normal 1.7-5.1]). The patient with the highest Co concentration $(71 \mathrm{nmol} / \mathrm{L})$ had an hematocrit of $91 \%$. The study also demonstrated that hematocrit values in these patients were positively correlated with the plasma levels of Co. However, these findings were not confirmed in another study performed in the same city.

It is interesting that $\mathrm{Cu}$ and $\mathrm{Zn}$ inhibit the activity of enzymes known as HIF prolil 4-hidroxylases (HPHs). The HPHs constitute important regulators of the homeostasis of oxygen in the organism. These enzymes determine the stability and activity of the Hypoxia Inducible Factor (HIF), a transcription factor that plays a fundamental role in the response of the organism to hypoxia, regulating the expression of a great number of genes. One of them is the erythropoietin (Epo) gene, which constitutes the hormonal stimulus for the increase in red blood cell production as a response to hypoxia.

It is possible then, that besides physiological and genetic factors, high concentrations of these metals in the blood of settlers of mining cities may constitute an important factor which contributes to EPO secretion stimulus, and therefore to excessive erythrocytosis and eventual development of CMS. 


\section{Prevention and treatment of CMS}

In recent times, increasing attention is being paid to different aspects that may be involved in the causal chain of CMS and were previously overlooked, including genetic predisposition, epigenetic influences on gene expression, and the role of environmental modifying factors that may be interacting with chronic hypoxia.

There is now clear evidence that different populations and individuals have different genetic susceptibilities to CMS constituting thus a potential area for designing and implementing preventive and therapeutic interventions, such as the possible use of genetic markers for screening purposes.

Also, several environmental factors such as early prenatal or postnatal exposure to different agents may modify gene expression and therefore the risk for developing CMS. It has been specifically suggested that perinatal factors may participate in the programming for later development of CMS. If confirmed, this may pave the way for early preventive interventions encompassing pregnancy and infancy.

Modification of life styles, dietary habits, reduction of indoor and outdoor pollution, and reduction of obesity and chronic respiratory diseases may modify the risk of developing CMS. The impact of these potential interventions must be thoroughly assessed.

However, before recommending the implementation at scale of preventive interventions, we need to perform rigorous studies on the epidemiology of CMS, in order to determine with precision the role of the postulated risk factors: Well-designed cohort studies will be particularly important.

Regarding treatment, there are several potential targets in the pathophysiological sequence of CMS that are potentially amenable to modification through nonpharmacological and pharmacological interventions. In brief, we could aim to inhibit the eryhtropoyetic response to reduce the increased red cell mass and thus the blood hyperviscosity, or we could aim to stimulate directly or indirectly the hypoxic ventilatory response through pharmacologic agents.

A variety of agents that includes methylxanthines, adrenergic blockers and dopaminergic antagonists, medroxyprogesterone, and peripheral ventilator stimulants such as almitrine, basically experimental, have not been rigorously tested through carefully designed randomized controlled trials.

Although used for a long time, there are no clinical trials on the efficacy and safety of phlebotomy with or without isovolemic hemodilution. Problems that render it problematic include invasiveness, practicality, acceptance, and transient effects. Isovolemic hemodilution is said to be better tolerated, as it may avoid hypovolemia.

Descent to low altitudes is the ideal treatment and leads to prompt improvement of signs and symptoms and reduction of excessive erythrocytosis. However, very often patients con not descent for economic, personal and family reasons.

Enalapril was studied in a small open randomized controlled trial in 26 Bolivian natives with altitude polycythemia (packed cell volume equal or above $55 \%$ ) and persistent proteinuria. Although packed red cell volume and proteinuria were significantly reduced over 2 years follow-up, there are several methodological concerns on this trial. The sample size was very small. The study was open to investigators and subjects and thus several relevant clinical endpoints and features related to quality of life could not be measured. Enrolled subjects had lower hematocrits than those included in other studies in Peru. Although renal disfunction has been described in CMS patients, the proportion of those with persistent proteinuria is not known and it may have been a source of selection bias.

Acetazolamide has been studied in randomized, placebo-controlled trials in the Peruvian Andes20 23). Although the first study included a relatively small number of subjects and 3 weeks follow-up, the second one followed patients for up to 6 months. Improvement of excessive erythrocytosis and ventilatory response were encouraging in these studies.

Due to the chronic relapsing nature of CMS, it is clear that both enalapril and acetazolamide need further large non-inferiority clinical trials that assess their comparative efficacy on several years of follow-up. 


\section{Conclusions and the way forward}

CMS is a multifactorial condition characterized by the presence of excessive hypoxemia and excessive erythrocytosis. Studies done among high altitude Andean natives showed an age-related decline in ventilation. Therefore, a failure of the hyperventilatory capacity and/or inefficient oxygenation of the blood may be the underlying pathophysiological cause of excessive hypoxemia. When the hypoxic condition is severe, is accompanied by pulmonary arterial hypertension, leading in advanced cases to right heart failure. Epidemiological studies, and the search for risk factors, confirm the presence of the underlying pathophysiological factors at work in CMS. Most of them are present in lesser degrees in apparently normal high altitude Andean natives and are more prominent in those with the clinical picture of CMS.

Overlapping cause and effect confounds a clear understanding of the pathophysiological mechanisms in CMS. For example, polycythemia, per se, presumed to be the consequence of hypoxemia, can by itself impair lung function. Similarly, the polycythemia resulting from increased EPO secretion, presumed to result from hypoxemia, can very likely itself lead to reduced kidney perfusion, which can further stimulate EPO secretion. Thus one of the intriguing aspects of this fascinating clinical syndrome is the unique opportunity it offers to analyze what is "adaptive" and what is not.

There is great variability in susceptibility, so one of the challenges is to explain not only the causes for its signs and symptoms, but also why at any given altitude some individuals are more likely to develop CMS than others. In addition to the physiological variables that lead to the pathophysiological sequence of CMS, other etiologic factors, of genetic origin, must also be considered. Studies at the molecular level should follow, looking for the genetic and molecular basis of the disease, i.e., the variability in HVR and EPO response, hemoglobin levels, etc. Furthermore, studies should be undertaken at the microvascular level to understand the patterns of $\mathrm{O} 2$ distribution in vessels of various sizes and the distribution of hematocrit and viscosity in these vessels.
In general there is a need to learn more about living, getting sick and getting older in the high altitude areas and CMS has provided the opportunity to expand our knowledge about the mechanisms found at different levels of acclimatization to high altitude. We have to continue research on this area as well as looking for better preventing and therapeutic alternatives.

\section{References}

1) Penaloza D, Sime F. Chronic cor pulmonale due to loss of altitude acclimatization (chronic mountain sickness). Am J Med 1971;50:728-743.

2) Antezana AM, Antezana G, Aparicio O, et al. Pulmonary hypertension in high-altitude chronic hypoxia: response to nifedipine. Eur Respir $J$ 1998;12:1181-1185.

3) Anand IS, Wu T. Syndromes of subacute mountain sickness. High Alt Med Biol 2004;5:156-170.

4) Sui GJ, Liu YH, Cheng XS, et al. Subacute infantile mountain sickness. J Pathol 1988;155:161-170.

5) Monge, M. Sobre un caso de Enfermedad de Váquez. Comunicación presentada a la Academia Nacional de Medicina., Lima 1925. 1-6.

6) Monge M. La enfermedad de los Andes. Anales de la Facultad de Medicina 1928:1-309.

7) León-Velarde F. et al., Consensus statement on chronic and subacute high altitude diseases. High Alt Med Biol. 2005; 6(2): 147-57.

8) Monge M, Monge CC. High Altitude Disease: Mechanism and Management. Springfield, Ill: Charles C. Thomas; 1966

9) León-Velarde F, Arregui A, Vargas M, et al. Chronic mountain sickness and chronic lower respiratory tract disorders. Chest 1994;106:151-155.

10) Winslow RM, Monge CC, Statham NJ, et al. Variability of oxygen affinity of blood: human subjects native to high altitude. J Appl Physiol 1981;51:1411-1416.

11) Monge CC, León-Velarde F, Arregui A. Increasing prevalence of excessive erythrocytosis with age among healthy high-altitude miners. $N$ Engl $J$ Med 1989;321:1271.

12) León-Velarde F, Arregui A, Monge C, et al. Aging at high altitudes and the risk of Chronic Mountain 
Sickness. J Wild Med 1993;4:183-188.

13) Ou LC, Sardella GL, Leiter JC, et al. Role of sex hormones in development of chronic mountain sickness in rats. $J$ Appl Physiol 1994;77:427-433.

14) León-Velarde F, Ramos MA, Hernandez JA, et al. The role of menopause in the development of chronic mountain sickness. Am J Physiol 1997;272: R90-94.

15) León-Velarde F, Rivera-Chira M, Tapia R, et al. Relationship of ovarian hormones to hypoxemia in women residents of 4,300 m. Am J Physiol Regul Integr Comp Physiol 2001;280:R488-493.

16) Guenard H, Vargas E, Villena $M$, et al. [Hypoxemia and the hematocrit in pathologic polycythemia due to altitude]. Bull Eur Physiopathol Respir 1984;20:319-324.

17) Reategui LL. Soroche cronico: observaciones realizadas en el Cuzco en 30 casos. Rev Agrup Med Amauta (Cusco) 1965;1:7-15.

18) Gonzales GF, Guerra-Garcia R. Niveles de hemoglobina en residentes de Cerro de Pasco $(4200 \mathrm{~m})$ según procedencia, actividad y edad. Acta de las Primeras Jornadas de Medicina y Cirguia de Altura 1978:1666-1171.

19) Santolaya BR, Arraya CJ, Vecchiola DA, et al. Gases y $\mathrm{pH}$ en sangre arterial en 176 hombres y 162 mujeres sanas trabajadores no mineros residentes a $2899 \mathrm{mts}$ de altura. Rev Hosp Roy H Glover 1982;2:7-18.

20) Rivera-Ch M, Huicho L, Bouchet $P$, et al. Effect of acetazolamide on ventilatory response in subjects with chronic mountain sickness. Respir Physiol Neurobiol 2008;162:184-189.

21) Monge CC, Leon-Velarde F. Physiological adaptation to high altitude: oxygen transport in mammals and birds. Physiol Rev 1991;71:1135-1172.

22) Maignan $M$, Rivera-Ch $M$, Privat $C$, et al. Pulmonary pressure and cardiac function in chronic mountain sickness patients. Chest 2009;135:499-504.

23) Monge CC, Leon-Velarde F. El Reto Fisiológico de Vivir en los Andes. Lima: IFEA, UPCH; 2003 